\title{
Xenografts derived from patients with head and neck cancer recapitulate patient tumour properties
}

\author{
HARUNA MAKITA $^{1}$, KAZUHIRA ENDO ${ }^{1}$, YOSHIYA KASAHARA ${ }^{1}$, ASUKA NAKATA $^{2}$, \\ MAKIKO MORIYAMA-KITA ${ }^{1}$, KAZUYA ISHIKAWA ${ }^{1}$, TAKAYOSHI UENO ${ }^{1}$, YOSUKE NAKANISHI ${ }^{1}$, \\ SATORU KONDO $^{1}$, NAOHIRO WAKISAKA ${ }^{1}$, NORIKO GOTOH ${ }^{2}$ and TOMOKAZU YOSHIZAKI ${ }^{1}$ \\ ${ }^{1}$ Department of Otolaryngology-Head and Neck Surgery, Graduate School of Medical Science, \\ Kanazawa University, Kanazawa, Ishikawa 920-8640; ${ }^{2}$ Department of Cancer Cell Biology, \\ Cancer Research Institute, Kanazawa University, Kanazawa, Ishikawa 920-1192, Japan
}

Received April 22, 2020; Accepted February 1, 2021

DOI: 10.3892/ol.2021.12646

\begin{abstract}
Rodent models mimic the heterogeneity of head and neck cancer (HNC) malignancies and are used to investigate $\mathrm{HNC}$-associated biomarkers and evaluate drug responses. To assess the utility of patient-derived xenografts (PDXs) as an HNC model, 18 tumour samples were obtained from surgical specimens of patients with HNC and implanted into non-obese diabetic severe combined immunodeficient mice. The histological features of PDXs and corresponding patient samples were compared. Furthermore, the present study investigated how PDX responses to anticancer drugs mimic patient clinical responses, as well as the expression of adenosine triphosphate-binding cassette transporters through chemotherapy in an HNC-PDX model. A total of five PDXs from patients with HNC exhibiting high correspondence with histopathological features of the original patient samples were established (establishment rate, 28\%). The responses of three PDXs to cisplatin were associated with clinical responses of the patients. ABC transporter expression was augmented in one PDX model after anticancer drug treatment, but not in PBS-treated passaged PDXs. PDX models exhibited similar biological
\end{abstract}

Correspondence to: Dr Kazuhira Endo, Department of Otolaryngology-Head and Neck Surgery, Graduate School of Medical Science, Kanazawa University, 13-1 Takaramachi, Kanazawa, Ishikawa 920-8640, Japan

E-mail: endok@med.kanazawa-u.ac.jp

Abbreviations: HNC, head and neck cancer; PDX, patient-derived xenograft; CDX, cell line-derived xenograft; $\mathrm{ABC}$ transporter, adenosine triphosphate-binding cassette transporter; NOD-SCID mouse, non-obese diabetic severe combined immunodeficient mouse; MDR-1, multiple drug resistance-1; MRP-2, multidrug resistance-associated protein-2; CCRT, concurrent cisplatin-radiation therapy

Key words: head and neck cancer, patient-derived xenografts, ABC transporters, MDR-1, MRP-2 and chemosensitive characteristics to those of the primary tumours. PDXs could be a useful preclinical tool to test novel therapeutic agents and identify novel targets and biomarkers in HNC.

\section{Introduction}

During the treatment of head and neck cancer (HNC), chemotherapy plays an important role, in addition to surgery and radiotherapy. However, the response rate to current drugs is not sufficient. To overcome this, the development of new, more effective anti-cancer agents and proper preclinical animal models to recapitulate patient disease is required. Rodent models have been conventionally used for translational cancer research, which ranges from the biological understanding of HNC to the evaluation of pharmacokinetics. Cell line-derived xenograft (CDX) models have been established by injecting cell lines, which were generated using tumour cells isolated from patients with HNC, subcutaneously into immunodeficient mice, and these are widely used for in vivo experiments. However, even though CDX models can suggest effective novel drug candidates, a significant number of these drugs fail in clinical trials, especially those for solid tumours (1). This limited predictive power is attributed to the CDX models' inability to capture the diverse heterogeneity of human malignancies as well as their differences from the actual patient tumours (2). To overcome the limitations of CDX models, patient-derived xenograft (PDX) models have been introduced to reflect the original patient tumours (2). PDX models have been established by transplanting tumour specimens directly into immunodeficient mice, and these retain the tumour heterogeneity observed in primary tumour specimens. In previous reports, PDX models of HNC were shown to recapitulate the histology of the original tumour and generate stable gene expression patterns (3); however, the therapeutic responses of HNC PDXs relative to those of the corresponding patient tumours have not been sufficiently evaluated. Here, we aimed to confirm that PDX models of HNC accurately replicate clinical outcomes for patients. To investigate the change in biomarker expression 
by administration of a target drug in a PDX model, we evaluated the expression of adenosine triphosphate-binding cassette $(\mathrm{ABC})$ transporters, a group of membrane transporters that translocate different molecules through the cellular membrane, mainly to the extracellular space, using ATP as an energy source (4). The expression levels of ABC transporters on exposure to anti-HNC drugs have been evaluated in vivo using HNC cell lines (5). Moreover, previous studies showed that ABC transporters are involved in intrinsic and acquired drug resistance and are associated with worse prognosis for HNC (6). Thus, in this study, we also assessed the relationship between ABC transporter expression and chemosensitivity in PDXs that reflect original patient tumours.

\section{Materials and methods}

Patient samples. Eighteen resected HNC tumour specimens obtained at the Division of Otolaryngology and Head and Neck Surgery at Kanazawa University were implanted for the establishment of PDXs. TNM classification of the patients was compliant with the UICC TNM classification, 8th edition (7). This study complied with the Declaration of Helsinki and was approved by the investigational Review Board of Kanazawa University (no. 2015-125). All patients included in this study provided written informed consent.

Establishment and passage of patient-derived xenografts. Non-obese diabetic severe combined immunodeficient (NOD-SCID) mice (Charles river laboratories Japan, INC., Kanagawa, Japan) were used to implant tumour fragments from patients (F0 generation). Five tumour pieces (1-2 mm in diameter) were suspended in Matrigel and subcutaneously transplanted into NOD-SCID mice within $24 \mathrm{~h}$ of tumour excision. Tumour fragments were implanted into $\sim 4$ mice on the basis of patient tumour volume. Additional tissue samples were stored at $-80^{\circ} \mathrm{C}$ with Cell Reserver One (Nacalai Tesque) and DMSO for further experiments. About 2-3 months post-transplantation, engrafted tumours of approximately $1 \mathrm{~cm}^{3}$ corresponding to $\mathrm{F} 1$ generations were surgically excised and small fragments were retransplanted into another NOD-SCID mouse. Xenograft tumours larger than $1 \mathrm{~cm}^{3}$ were not observed in this study. Tumours were passaged no more than five times. Tumour collection date $[\mathrm{X}($ year $) /($ month $) /($ day) $]$, time to harvest (days), and last passage are shown in Tables SI, SII, and SIII. All animal procedures were approved by the Ethical Committee of the Laboratory for the Animal Experiments, Graduate School of Medical Science, Kanazawa University (permit number: AP-173861) and were performed in compliance with the guidelines of this committee. A flow rate of $30 \%$ chamber volume displaced/min with $\mathrm{CO}_{2}$ was used for euthanizing the animals that had completed the experiment.

Hematoxylin and eosin $(H \& E)$ staining of primary tumours and xenografts. To compare xenografts to the original specimens, tissues from patient tumours and PDXs were formalin-fixed immediately after collection, paraffin-embedded, and stained with $\mathrm{H} \& \mathrm{E}$ according to standard protocols.
Short tandem repeat (STR) profiling. The profiles of 10 core STR markers (TH01, D21S11, D5S818, D13S317, D7S820, D16S539, CSF1PO, AMEL, vWA, and TPOX) were examined to determine the relatedness of patient tumours to a series of their PDXs (BEX Co., Ltd.). All frozen tissue specimens were used in this experiment.

Immunohistochemistry (IHC). The original patient tumours and PDXs were embedded in paraffin and used for the immunohistochemical analysis of the expression of $\mathrm{Ki}-67$, epidermal growth factor receptor (EGFR), p53, multiple drug resistance-1 (MDR-1), and multidrug resistance-associated protein-2 (MRP-2). MDR-1 and MRP-2 are ABC transporters that were reported to be prognostic markers for HNC (6). Three-micrometer-thick sections were prepared from each block of tissues embedded in paraffin. Deparaffinized sections were treated with $3 \%$ hydrogen peroxide for $10 \mathrm{~min}$ to inactivate endogenous peroxidase activity. The sections were then incubated with a protein blocker (Dako) for $20 \mathrm{~min}$ and incubated at $4^{\circ} \mathrm{C}$ overnight with anti-Ki-67 (rabbit monoclonal, ab16667, RRID:AB_302459, 1:200, Abcam), anti-EGFR monoclonal (rabbit monoclonal, ab40815, RRID:AB_732110, 1:250, Abcam), anti-p53 monoclonal (rabbit monoclonal, ab33889, RRID:AB_776988, 1:200, Abcam), anti-MDR-1 polyclonal (rabbit polyclonal, bs-0563R, RRID:AB_10856233, 1:100, BIOSS Inc., Boston, USA), and anti-MRP-2 polyclonal (rabbit polyclonal, bs-1092R, RRID:AB_10856413, 1:100, BIOSS Inc) primary antibodies. The sections were then washed three times with phosphate-buffered saline (PBS, pH 7.2). After washing, the sections were exposed to Envision + System-HRP Labelled Polymer Anti-Rabbit secondary antibody (Dako) for $60 \mathrm{~min}$. The reaction products were developed by immersing the sections in a 3,3'-diaminobenzidine tetrahydrochloride solution. Sections were counterstained with hematoxylin. The $\mathrm{Ki}-67$ index is calculated as the percentage of positive cells per 1,000 counts of the total cells.

Chemosensitivity testing. F2 generation PDXs obtained from NOD-SCID mice were engrafted into BALB/c-nu/nu mice (Charles River Laboratories, Inc.), and small fragments were retransplanted into new BALB/c-nu/nu mice for drug administration tests. When tumours were palpable, F3 generation PDX tumour-bearing BALB/c-nu/nu mice were randomized to treatment or control groups consisting of six mice each. BALB/c-nu/nu mice were chosen because passaged tumours would continue to grow in less immunocompromised mouse strains and to ensure the comparability of results because the appropriate doses were previously assessed in this strain by our group. PDX tumour-bearing BALB/c-nu/nu mice were treated for two consecutive weeks with weekly paclitaxel (20 mg/kg; Nippon Kayaku Co., Ltd.), weekly cisplatin (2.5 mg/kg; Nippon Kayaku), or weekly PBS (as a control) intravenous injections. Two-dimensional tumour measurements were performed with a sliding calliper once weekly. Individual tumour volumes were calculated using the formula: $\mathrm{V}\left(\mathrm{mm}^{3}\right)=1 / 2 \mathrm{xaxb}^{2}$ (where ' $\mathrm{a}$ ' was the longest tumour diameter and ' $b$ ' was the shortest tumour diameter). We could test drug response of $\mathrm{P}-2, \mathrm{P}-3$ and $\mathrm{P}-5$ in vivo, but not of $\mathrm{P}-1$ and $\mathrm{P}-4$, because we only had frozen PDX specimens of P-1 and 
P-4 and could not make PDX models for this examination from these samples.

Scoring of ABC transporter protein expression. The sections were observed using the ECLIPSE Ni upright microscope (Nikon). Positive ratio scores were based on the percentage of positive cells in a high-power field, as follows: Score 0 (0-10\%); score $1(11-25 \%)$; score 2 (26-50\%); score 3 (51-75\%); and score 4 (76-100\%). The investigator also ranked the expression intensity from 0 (no expression) to 3 (very strong expression). The positive ratio score and expression intensity score were multiplied, and each tumour score was then calculated as the mean of five high-power fields. For F3' (F3 after drug treatment), the final scores were averaged from six mice. Consequently, the lowest score was 0 , and the highest score was 12 . Tissues slides from healthy liver sections were used as positive controls. We could examine the expression of $\mathrm{ABC}$ transporters expression in $\mathrm{P}-2, \mathrm{P}-3$ and $\mathrm{P}-5$, not $\mathrm{P}-1$ and $\mathrm{P}-4$, as mentioned above.

Statistical analysis. All analyses were performed with SPSS statistical software (Version 23; RRID:SCR_002865, IBM Corp.). Inter-relations between engraftment rates and patient characteristics were examined by performing Fisher's exact tests or unpaired Student's t-tests. The in vivo effects of paclitaxel or cisplatin vs. the control were evaluated using a mixed two-way analysis of variance with Bonferroni's test as a post hoc test to compare the tumour volumes. The protein expression scores of MDR-1 and MRP-2 in F0, F3, and F3' (F3 at the end of chemotherapy) groups were evaluated by performing a Kruskal-Wallis test, using Mann-Whitney tests and a Bonferroni correction as a post hoc test [F3 or F3' (treated with PBS, paclitaxel, and cisplatin) vs. F0]. A P-value of $<0.05$ was considered statistically significant.

\section{Results}

Establishment of PDXs and patient characteristics. The primary lesions of tumours are shown in Table I. Oral cancers comprised six cases and were the most common. Of all 18 resected HNC tumours that were implanted into NOD-SCID mice for PDX establishment, five (28\%) engrafted and could be passaged. The characteristics of patients are shown in Table II. PDX establishment was significantly associated with surgical margin-positive cases, but not with sex, smoking status, pre-treatment, histologic type (squamous cell carcinoma or other), Ki-67 index, TNM, staging, or recurrence at 6 months after surgery. When surgical margin-positive cases were retrospectively evaluated from the pathological report, extranodal infiltration was identified in three PDX-failed cases and in two PDX-established cases; excision margin positive primary lesions were identified in two PDX-failed cases and in three PDX-established cases. The positivity of infiltration features (e.g. vein, lymphatic, and perineural invasion) was also determined according to the pathological report. Because 3 of 5 PDX-succeeded cases did not have any information regarding infiltration patterns, the sample number included in our study was too small to perform any statistical analysis. Moreover, all five
Table I. Primary tumour lesions.

Primary lesion

Patients, n $(\mathrm{n}=18)$

\begin{tabular}{ll}
\hline Oral cancer & 6 \\
Tongue & 4 \\
Floor of mouth & 1 \\
Gums & 1 \\
Hypopharynx & 3 \\
Oropharynx & 2 \\
Larynx & 2 \\
Salivary gland & 2 \\
Paranasal Sinus & 1 \\
Nasopharynx & 1 \\
Thyroid (papillary carcinoma and & 1 \\
squamous cell carcinoma) & \\
\hline
\end{tabular}

patients from whom PDX tumours were developed were treated with concurrent cisplatin radiation therapy (CCRT) after surgery. The detailed patient profiles accompanying the five models (P-1, P-2, P-3, P-4, and P-5) are shown in Table III. P-1 and P-2 were cisplatin-non-responder PDX models according to the definition of recurrence within 6 months post-CCRT. In contrast, P-3, P-4, and P-5 were cisplatin-responder PDX models according to the definition of recurrence 6 months or later or complete remission for more than 6 months post-CCRT. Further, the H\&E staining of primary tumours and xenografts is shown in Fig. 1. The tissue type and degree of differentiation were consistent between primary tumours and xenografts. The histopathologic features of human HNC tumours were also retained in mice. The Ki-67 index was passed down from all patient samples to the PDXs (Table SIV). We confirmed the uniqueness between the patient tumours and each PDX (P-1, P-3, P-4, and P-5) or two generations of PDXs (P-2) using STR profiling (Figs. 2 and 3; Table IV). Except P-1, which had an evaluation value $(\mathrm{EV})$ of $0.53[\mathrm{EV}=$ (number of coincidental peaks) $\times 2 /($ total number of peaks in tissue $\mathrm{A}+$ total number of peaks in tissue B)], all sample pairs showed an $\mathrm{EV}$ of 0.8 or higher, indicating that the tissues were almost identical (8).

Chemosensitivity testing. To compare chemotherapy efficacies between patients and PDX models, chemosensitivity testing was performed using three models (P-2, P-3, and P-5). For P-2, bone metastasis appeared 2 months post-CCRT, and thus, this case was considered a cisplatin-non-responder. For P-3 and P-5, complete remission continued for 6 months post-CCRT; therefore, these cases were considered cisplatin-responders. The tumour growth curves for these models are shown in Fig. 4. For P-3, cisplatin and paclitaxel treatment resulted in significant decreases in the tumour sizes at days 35 and 42 compared with those in the control (PBS). For P-5, cisplatin and paclitaxel treatment resulted in significant decreases in the tumour sizes at day 42 compared with those in the control (PBS). In contrast, for P-2, a cisplatin non-responder HNC 
Table II. Patient characteristics.

\begin{tabular}{|c|c|c|c|c|}
\hline Characteristics & $X G(n=5)$ & No $X G(n=13)$ & Total $(\mathrm{n}=18)$ & P-value \\
\hline Age, years (mean; range) & $58.4(43-69)$ & $65.9(53-84)$ & & 0.096 \\
\hline Sex, $n$ & & & & 0.522 \\
\hline Male & 5 & 10 & 15 & \\
\hline Female & 0 & 3 & 3 & \\
\hline Smoking status, $\mathrm{n}$ & & & & 0.533 \\
\hline Yes & 3 & 11 & 14 & \\
\hline No & 2 & 2 & 4 & \\
\hline Origin, $\mathrm{n}$ & & & & 0.583 \\
\hline Primary & 3 & 10 & 13 & \\
\hline Lymph node & 2 & 3 & 5 & \\
\hline Histology, $\mathrm{n}$ & & & & 0.278 \\
\hline $\mathrm{SCC}$ & 5 & 9 & 14 & \\
\hline Other & 0 & 4 & 4 & \\
\hline Surgical margin, $\mathrm{n}$ & & & & 0.036 \\
\hline Positive & 5 & 5 & 10 & \\
\hline Negative & 0 & 8 & 8 & \\
\hline Ki-67 index, \% (mean; range) & $7(5-10)$ & $5(0-25)$ & & 0.178 \\
\hline Postsurgical treatment, $\mathrm{n}$ & & & & 0.002 \\
\hline $\mathrm{CDDP}+\mathrm{RT}$ & 5 & 2 & 7 & \\
\hline Only RT & 0 & 3 & 3 & \\
\hline TS-1 & 0 & 2 & 2 & \\
\hline No treatment & 0 & 6 & 6 & \\
\hline Primary/recurrent, $\mathrm{n}$ & & & & 0.583 \\
\hline Primary & 3 & 10 & 13 & \\
\hline Recurrent & 2 & 3 & 5 & \\
\hline $\mathrm{T}, \mathrm{n}$ & & & & 0.596 \\
\hline T0-2 & 1 & 6 & 7 & \\
\hline T3-4 & 4 & 7 & 11 & \\
\hline $\mathrm{N}, \mathrm{n}$ & & & & $>0.999$ \\
\hline N0 & 2 & 7 & 9 & \\
\hline N1-3 & 3 & 6 & 9 & \\
\hline $\mathrm{M}, \mathrm{n}$ & & & & $>0.999$ \\
\hline M0 & 5 & 13 & 18 & \\
\hline M1 & 0 & 0 & 0 & \\
\hline Stage, $\mathrm{n}$ & & & & $>0.999$ \\
\hline I-II & 0 & 2 & 2 & \\
\hline III-IV & 5 & 11 & 16 & \\
\hline Recurrence at 6 months after surgery, $\mathrm{n}$ & & & & $>0.999$ \\
\hline Yes & 2 & 4 & 6 & \\
\hline No & 3 & 8 & 11 & \\
\hline Other $^{\mathrm{a}}$ & 0 & 1 & 1 & \\
\hline
\end{tabular}

${ }^{a}$ The patient died due to disease other than head and neck cancer prior to the 6-month mark following surgery. XG, PDX established; No XG, failed to form PDX; SCC, squamous cell carcinoma; CDDP, cisplatin; RT, radiation therapy; PDX, patient-derived xenograft.

PDX model, there were no significant differences in tumour size between the PBS and cisplatin-treated groups. For P-3 and P-5, the tumour size decreased significantly in the cisplatin group, increased significantly in the PBS group, and was not significantly different in the paclitaxel group based on day 28. On the contrary, for P-2, the tumour size was significantly increased in the cisplatin and PBS groups based on day 28 . 
Table III. Detailed profiles of patients in the five models.

\begin{tabular}{|c|c|c|c|c|c|c|c|}
\hline Tumour ID & $\begin{array}{l}\text { Site of } \\
\text { tumour origin }\end{array}$ & $\begin{array}{l}\text { Age, } \\
\text { years }\end{array}$ & Sex & Primary/recurrent & TNM, Stage & Histology & Progress after CCRT \\
\hline P-1 & Tongue & 43 & Male & Recurrent & $\begin{array}{l}\text { T4aN0M0, } \\
\text { Stage IVA }\end{array}$ & SCC & $\begin{array}{l}\text { Neck and armpit lymph node } \\
\text { metastases appeared at } 1 \text { month }\end{array}$ \\
\hline $\mathrm{P}-2$ & Hypopharynx (LN) & 68 & Male & Primary & $\begin{array}{l}\text { T2T2bM0, } \\
\text { Stage IVA }\end{array}$ & SCC & $\begin{array}{l}\text { Bone metastasis appeared at } \\
2 \text { months }\end{array}$ \\
\hline P-3 & Hypopharynx (LN) & 57 & Male & Primary & $\begin{array}{l}\text { T3N3M0, } \\
\text { Stage IVB }\end{array}$ & $\mathrm{SCC}$ & CR at 14 months \\
\hline P-4 & Laryngeal & 55 & Male & Recurrent & $\begin{array}{l}\text { T4aN2bM0, } \\
\text { Stage IVA }\end{array}$ & $\mathrm{SCC}$ & $\begin{array}{l}\text { Lung metastasis appeared at } \\
11 \text { months }\end{array}$ \\
\hline P-5 & Paranasal sinus & 69 & Male & Primary & $\begin{array}{l}\text { T4bN0M0, } \\
\text { Stage IVB }\end{array}$ & $\mathrm{SCC}$ & CR at 6 months \\
\hline
\end{tabular}

CCRT, concurrent chemoradiation therapy (cisplatin); LN, lymph node (neck metastasis); CR, complete remission; SCC, squamous cell carcinoma.

Table IV. STR profiling of tumour samples.

\begin{tabular}{|c|c|c|c|c|c|c|c|c|c|c|c|}
\hline Tumour samples & TH01 & D21S11 & D5S818 & D13S317 & D7S820 & D16S539 & CSF1PO & AMEL & vWA & TPOX & $\mathrm{EV}$ \\
\hline P-1 (F0) & 6,7 & $29,33.2$ & 9,13 & 11 & 10,12 & 10,12 & 7,12 & $\mathrm{X}, \mathrm{Y}$ & 16,17 & 8,11 & 0.53 \\
\hline P-1 (F3) & $6,9.3$ & 30 & 11 & 11 & 10,12 & 9 & 11 & $\mathrm{X}, \mathrm{Y}$ & 16,17 & 9,11 & \\
\hline P-2 (F1) & 7,9 & 31.2 & 13 & 11 & 11 & 9,11 & 11,12 & $\mathrm{X}, \mathrm{Y}$ & 14,17 & 8,10 & 1 \\
\hline P-2 (F3) & 7,9 & 31.2 & 13 & 11 & 11 & 9,11 & 11,12 & $\mathrm{X}, \mathrm{Y}$ & 14,17 & 8,10 & \\
\hline P-3 (F0) & 6,9 & 29,31 & 11,13 & 8,12 & 10,11 & 10 & 10,12 & $\mathrm{X}, \mathrm{Y}$ & 17 & 8,9 & 0.88 \\
\hline P-3 (F3) & 6,9 & 29,31 & 13 & 12 & 10,11 & 10 & 12 & $\mathrm{X}$ & 17 & 8,9 & \\
\hline P-4 (F0) & $6,9.3$ & 29,30 & 11,13 & 8,11 & 10,12 & 9 & 11,12 & $\mathrm{X}, \mathrm{Y}$ & 16,17 & 9,11 & 0.88 \\
\hline P-4 (F1) & $6,9.3$ & 30 & 11 & 11 & 10,12 & 9 & 11 & $\mathrm{X}, \mathrm{Y}$ & 16,17 & 9,11 & \\
\hline P-5 (F0) & 7,9 & 30 & 10,11 & 8,11 & 10,11 & 9,11 & 11,12 & $\mathrm{X}, \mathrm{Y}$ & 18 & 8,11 & 0.94 \\
\hline P-5 (F2) & 7,9 & 30 & 10,11 & 8,11 & 10,11 & 9,11 & 11 & $X$ & 18 & 8,11 & \\
\hline
\end{tabular}

TH01, D21S11, D5S818, D13S317, D7S820, D16S539, CSF1PO, AMEL, vWA, and TPOX are 10 core STR markers. Alleles are shown for each STR marker. The rightmost column shows the EV between the patient tumours and each PDX (P-1, P-3, P-4 and P-5) or two generations of PDXs (P-2). P-1, P-2, P-3, P-4, and P-5 are tumour IDs. F0, Patient tumour. F1, F2, and F3; generation (1, 2, and 3 passages). EV, evaluation value.

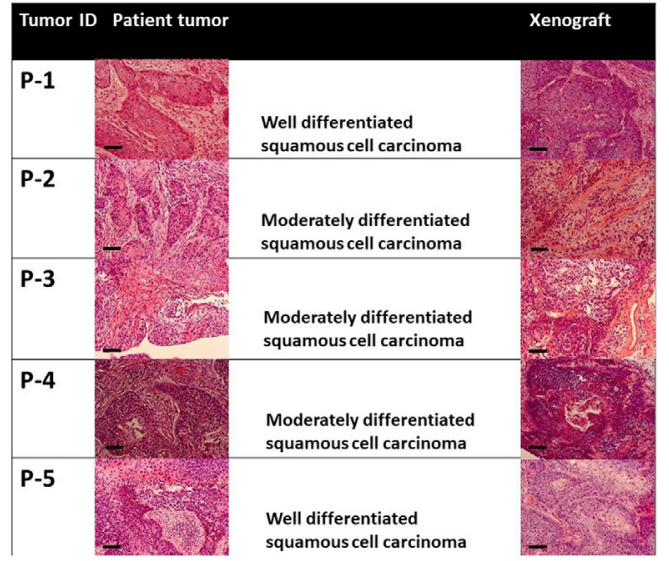

Figure 1. Histological features based on haematoxylin and eosin staining revealing excellent similarity between patient-derived xenografts and the corresponding head and neck tumours from patients. Scale bar, $100 \mu \mathrm{m}$. Magnification, x200. The xenografts are: P-1, F5 generation (5 passages); P-2, F3 generation; P-3, F3 generation; P-4, F1 generation; and P-5, F1 generation.
Evaluation of $A B C$ transporter response to chemotherapy. To evaluate the expression of ABC transporters, MDR-1 and MRP-2 levels were compared between tumours from drug-treated mice and patient samples (F0). The expression intensity scores are shown in Fig. 5. Averages of the final scores are shown in Fig. 6. In P-3, the protein expression scores of MDR-1 were 5.6 for F0, 7.8 for F3' (treated with cisplatin), and 10.4 for F3' (treated with paclitaxel), and the scores for MRP-2 changed from 5.6 for F0 to 10.4 for F3' (treated with cisplatin) and 8.85 for F3' (treated with paclitaxel). The scores after drug administration, except for those of MDR-1 in F3' (treated by cisplatin) samples, significantly increased compared with those in F0 samples for P-3. For P-5 and P-2, the protein expression scores in F3' (treated by paclitaxel and cisplatin) samples were not significantly different from those in F0 samples. In all three models, the MDR-1 and MRP-2 expression scores in F3 and F3' (treated by PBS) samples were not significantly changed compared with those in F0 samples. 

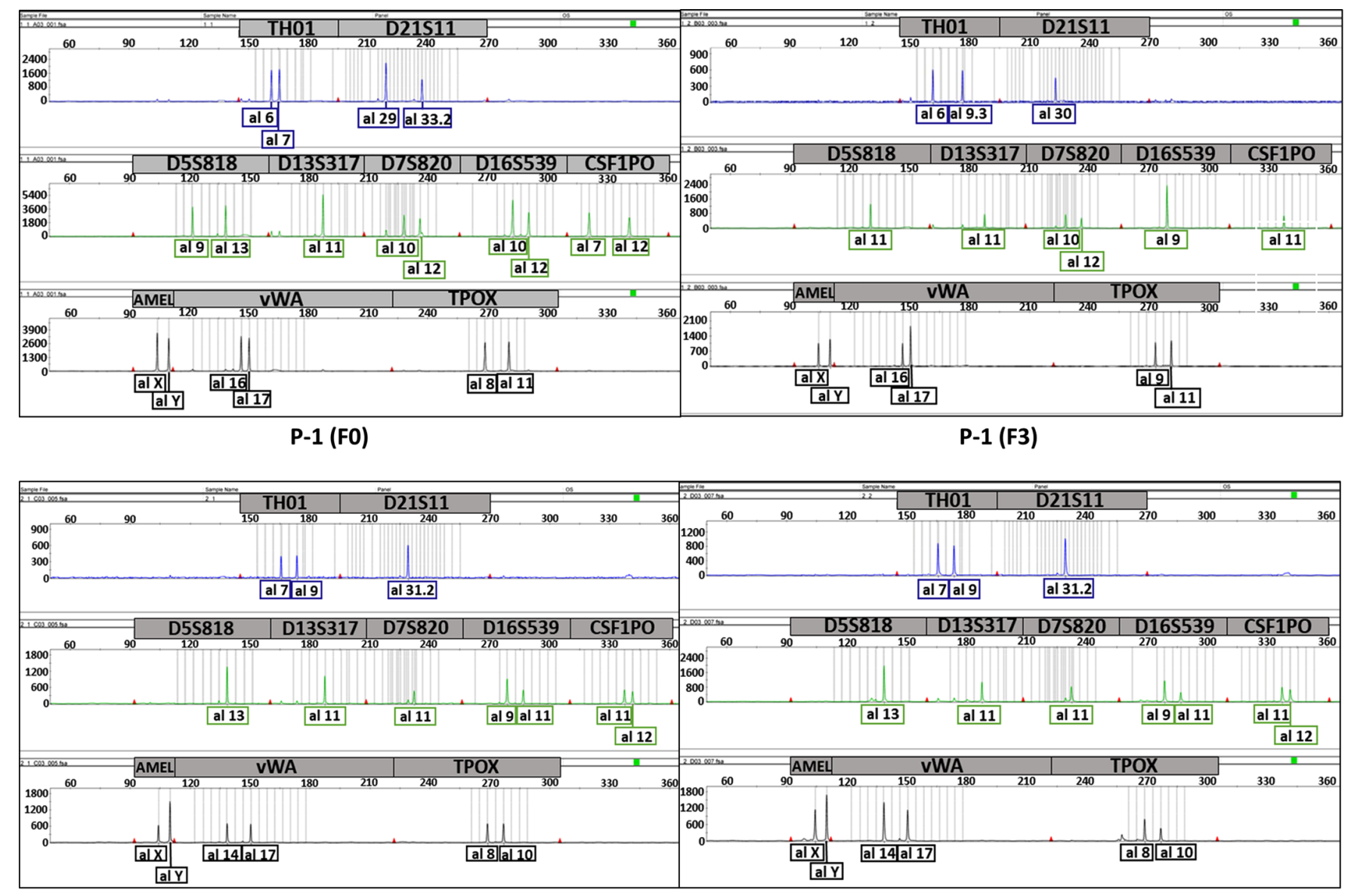

P-2 (F1)

P-2 (F3)

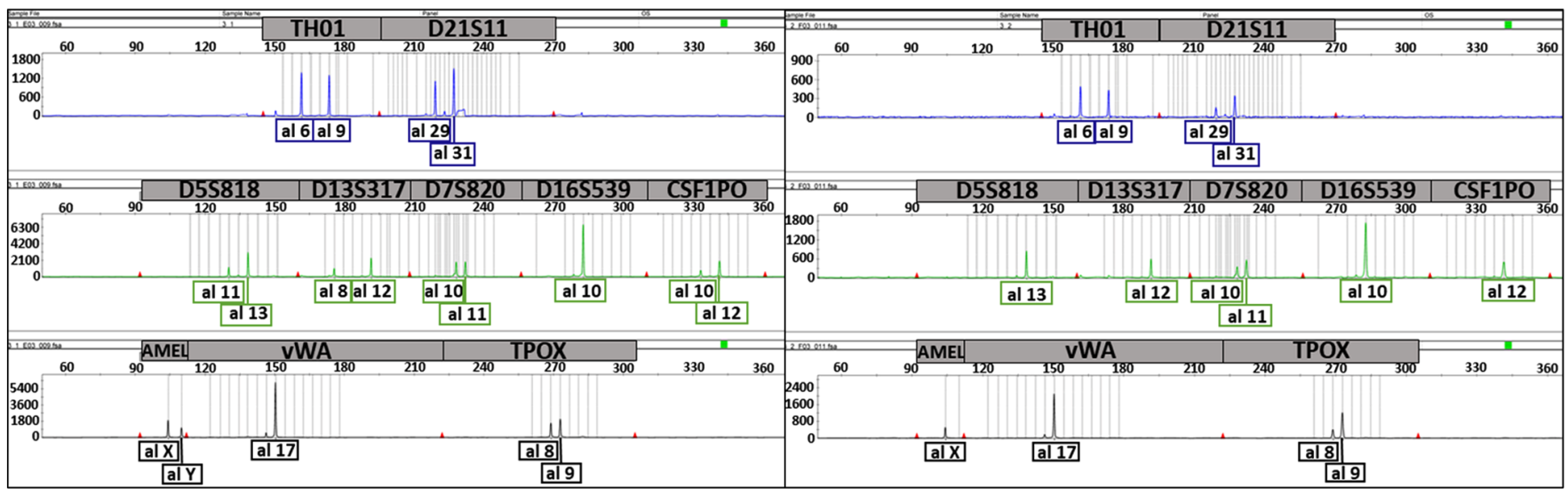

P-3 (F0)

P-3 (F3)

Figure 2. Electropherogram showing the unique profile of each tumour to compare patient tumours (F0) and their PDXs (P-1 and P-3) or different generations of each PDX to another (P-2). The top panel (blue) shows the graphs for TH01 and D21S11, the middle panel (green) shows the graphs for D5S818, D13S317, D7S820, D16S539 and CSF1PO, and the bottom panel (black) shows the graphs for AMEL, vWA and TPOX. P-1, F3 generation (3 passages); P-2, F1 and F3 generation; P-3, F3 generation; PDX, patient-derived xenograft.

\section{Discussion}

In this study, we established PDXs from the tumours of patients with HNC. H\&E staining, Ki-67 index, and STR profiling demonstrated the consistency between the patient tumours and xenografts. We showed the genomic stability of PDXs (P-2, P-3, P-4, and P-5) and identified genotypic changes through serial propagation (P-1). Further, the protein expression patterns of EGFR and p53 were passed down from all patient samples to the PDXs (Fig. S1). Although the sample size of this study was relatively small, our PDX models may be capable of recapitulating the complexity of HNC malignancy remarkably well. In cancers other than HNC, histologic and immunohistochemical analyses have already revealed a high degree of pathologic similarity (9). Of the 18 tumour samples obtained from surgical specimens, five (28\%) PDX models were established. The rate of PDX establishment was significantly associated with the presence of positive 


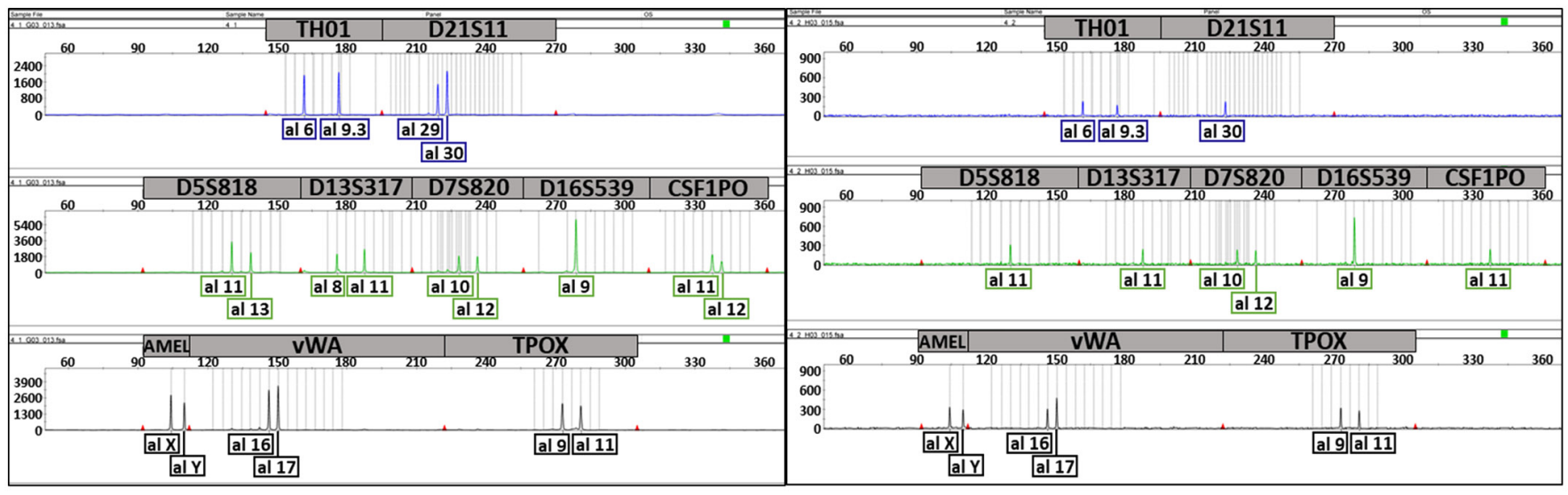

P-4 (F0)

P-4 (F1)

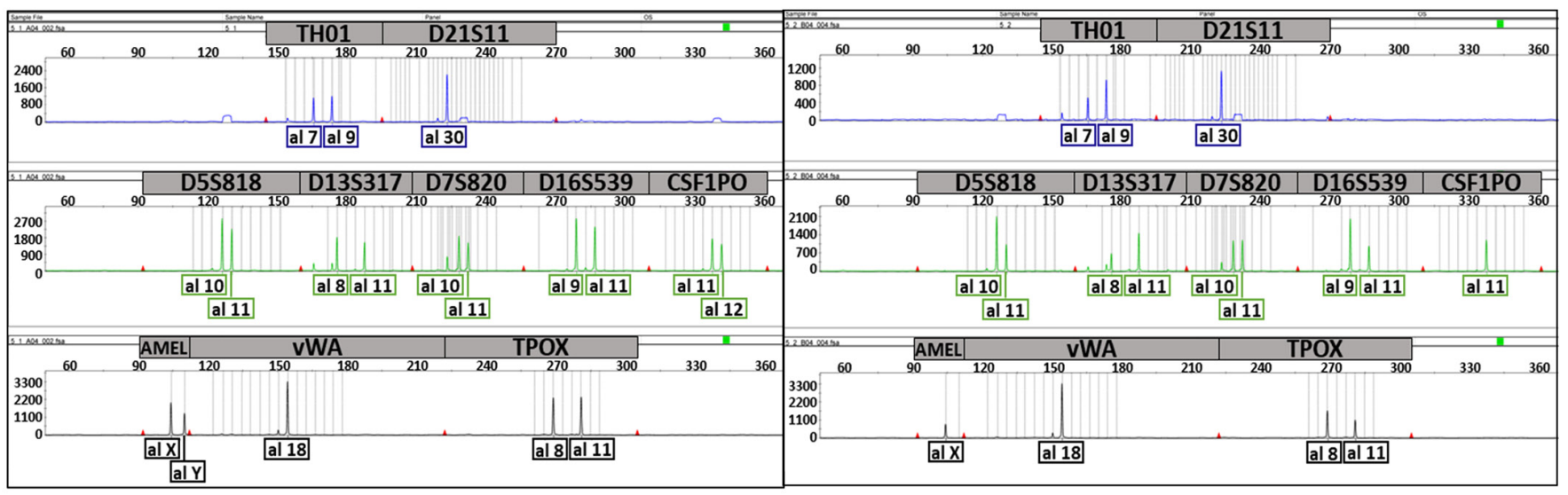

P-5 (F0)

P-5 (F2)

Figure 3. Electropherogram showing the unique profile of each tumour to compare patient tumours (F0) and their PDXs (P-4 and P-5). The top panel (blue) shows the graph for TH01 and D21S11, the middle panel (green) shows the graph for D5S818, D13S317, D7S820, D16S539 and CSF1PO and the bottom panel (black) shows the graph for AMEL, vWA and TPOX. P-4, F1 generation; and P-5, F2 generation. PDX, patient-derived xenograft.
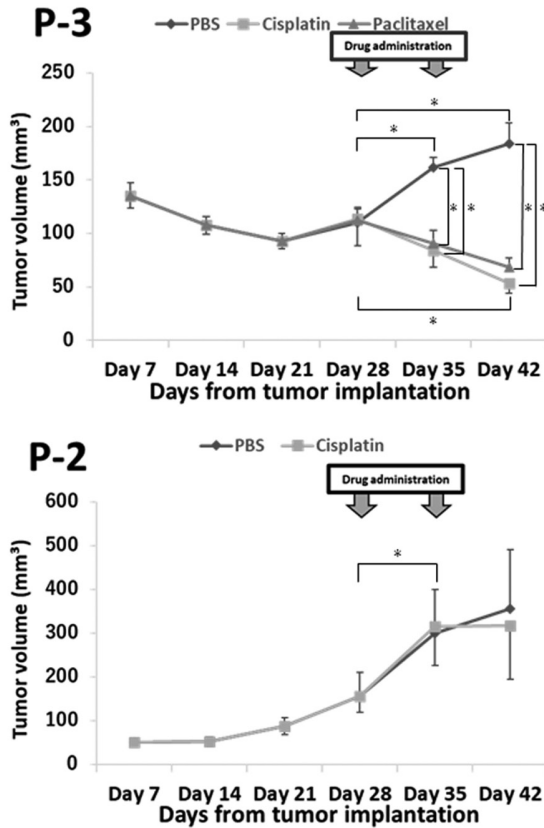

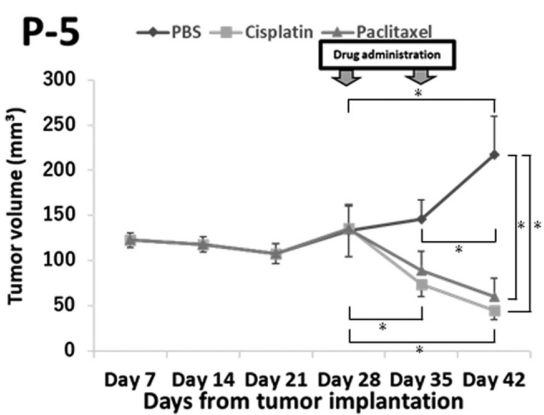

Days from tumor implantation

Figure 4. P-3 and P-5 PDXs derived from patients with head and neck cancer respond to cisplatin and those from P-2 do not. Tumours from patients were transplanted into non-obese diabetic severe combined immunodeficient mice and serially transplanted into new animals upon tumour growth. Xenografts were treated with cisplatin and tumour growth curves were compared. PDX models mirrored the patients' clinical responses to cisplatin. The error bars indicate the standard error. $\mathrm{P}<0.05$ was considered to indicate a statistically significant difference. ${ }^{*} \mathrm{P}<0.05$ as indicated. For comparisons by administration date (day 28 , 35 and 42), the PBS group was the control. For comparisons by drug (PBS, cisplatin and paclitaxel), day 28 was the control. PDX, patient-derived xenograft. 

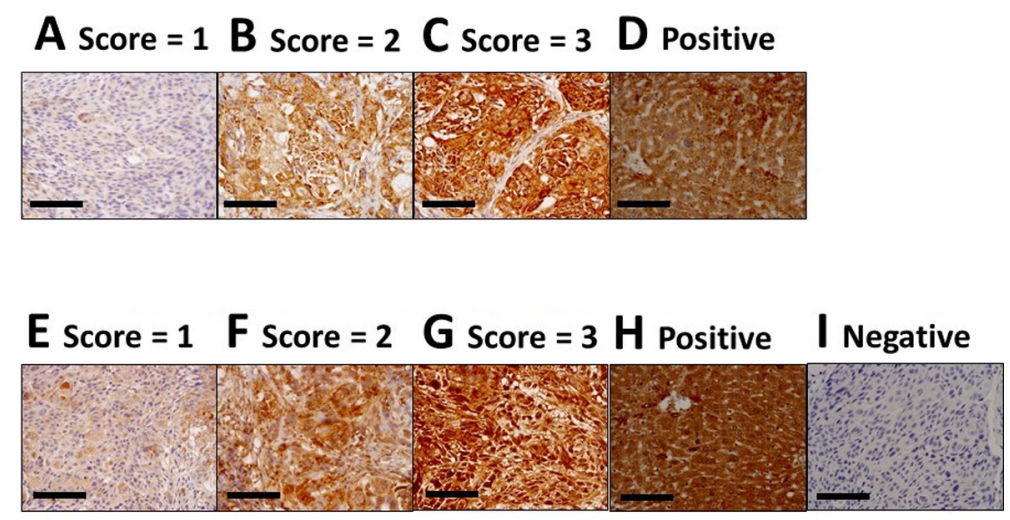

Figure 5. MDR-1 and MRP-2 immunostaining images. (A-D) Expression intensity scores of MDR-1 based on IHC staining. Intensity scores were (A) 1 , (B) 2 and (C) 3. (D) MDR-1 positive control. (E-H) Expression intensity scores of MRP-2 based on IHC staining. Intensity scores were (E) 1 , (F) 2 and (G) 3. (H) MRP-2 positive control. (I) Negative control (F0 of P-3, no primary control for secondary antibody). Scale bar, $100 \mu \mathrm{m}$. Magnification, x400. IHC, immunohistochemical; MDR-1, multiple drug resistance-1; MRP-2, multidrug resistance-associated protein-2.

A MDR-1 expression score
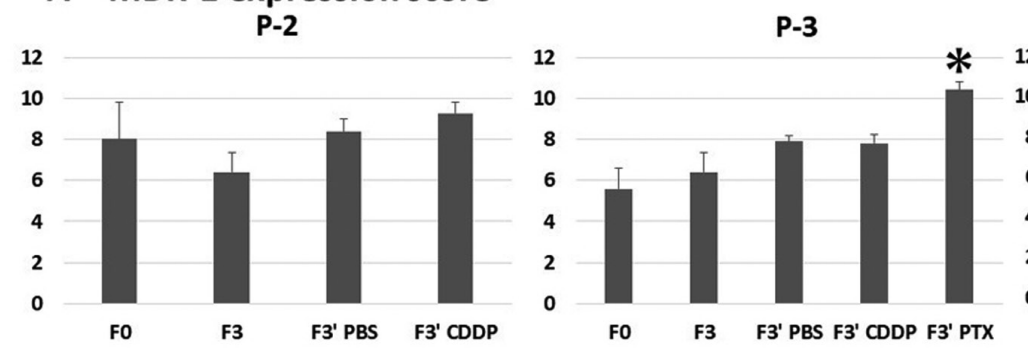

B MRP-2 expression score
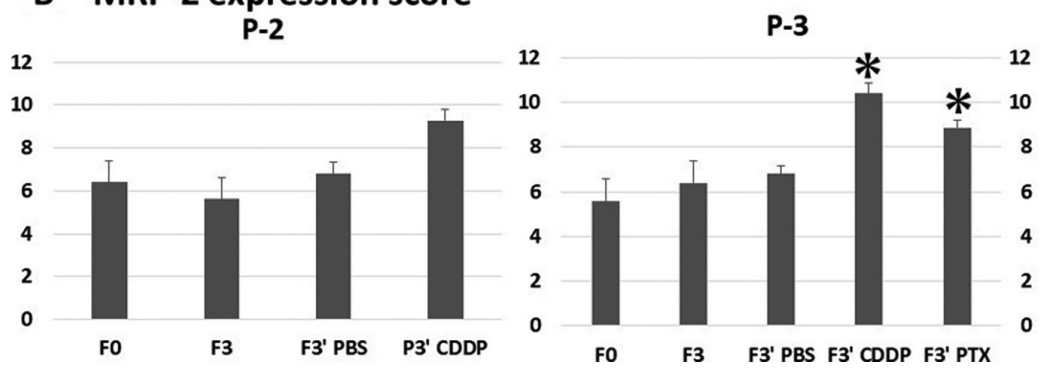

Figure 6. MDR-1 and MRP-2 expression score graphs. Average final (A) MDR-1 and (B) MRP-2 expression scores of F0 patients compared with those of other in vivo passage numbers. Tumours from patients were transplanted into non-obese diabetic severe combined immunodeficient mice and serially transplanted into new animals upon tumour growth. Immunohistochemistry was used to compare MDR-1 and MRP-2 expression among the groups. The error bars indicate the standard error. $\mathrm{P}<0.05$ was considered to indicate a statistically significant difference. ${ }^{*} \mathrm{P}<0.05$ vs. F0. CDDP, cisplatin; PTX, paclitaxel; PBS, phosphate buffered saline (control); MDR-1, multiple drug resistance-1; MRP-2, multidrug resistance-associated protein-2.

surgical margins and CCRT after surgery. Previous data regarding the engraftment rate of HNC PDXs suggest variability, from 17 to $85 \%(3,10,11)$. The success rates of PDX are influenced by several factors (2). First, the patient tumour characteristics might be related to the success rate. With regard to clinical parameters, PDX engraftment was previously found to be associated with poor disease free and overall survival (12) and an increased risk of metastasis (13). Histologically, lymphovascular invasion (14) and perineural invasion (15) are associated with increased PDX formation. Prince et al (16) reported that engraftment bias may occur because of the stem cell-like property (CD $44^{+}$cells). Theoretically, tumours with massive invasion have more frequent positive surgical margins. In this study, the positivity of infiltration features (e.g., vein, lymphatic, and perineural invasion) was determined based on the pathological records. We undertook statistical analysis for the case based on comments from the pathologists. However, more than half of the succeeded cases did not have any information regarding invasion patterns. It was thus inappropriate to perform statistical analysis for the entire cohort. Although we cannot mention the direct relationship between the positive surgical margins and PDX engraftment in this study alone, we suggest that patient tumour characteristics such as invasive ability or stem-cell like property around the tumour could be considered as factors that influence the success rates of PDX. Second, the degree of immunodeficiency in mice might influence the success rate. NOD-SCID mice do not contain functional T and B cells; however, NK cells are somewhat 
functional. In contrast, NOD/Scid/IL2R $\gamma$ null (NSG) mice do not have functional T, B, or NK cells (2). Therefore, NSG mice have recently become preferred, compared with other mouse strains, for the development of PDXs. Third, the location of implantation might affect the success rate (2). We implanted primary tumours subcutaneously, the most common site of implantation; however, implantation in the renal capsule has been found to maintain the original tumour stroma and the equivalent host stroma, making this approach more likely to succeed (2).

Although not yet investigated in $\mathrm{HNC}$, correlations have been observed in clinical outcomes between drug responses in PDXs and other organs of patients with cancer $(12,17)$. Our study also showed an association between the anti-tumour effect of cisplatin in three PDX models (P-2, P-3, and P-5) and that observed in corresponding patients. PDX tumour sizes in P-3 and P-5 models, for which a positive response to CCRT was noted in corresponding patients, were ultimately reduced to $29 \%(\mathrm{P}-3)$ and $21 \%(\mathrm{P}-5)$ of respective PBS-treated tumour sizes upon cisplatin treatment. In contrast, PDX tumour sizes in the P-2 model, derived from a tumour refractory to CCRT, showed no differences between PBS and cisplatin treatment groups. We considered the following two reasons for the rapid drug response in P-3 and P-5 PDX models. First, the rapid tumour reduction rate in PDX models reflected the chemosensitivity of the original tumour that was categorized as platinum-sensitive. In many clinical studies on recurrent/metastatic HNC, patients with recurrence after platinum-containing therapy within 6 months were platinum resistant and those after 6 months were platinum sensitive. This classification based on ovarian cancer studies $(18,19)$ was used in this study. Because the correlation of drug responses between the original tumours and PDXs in various cancers has been reported, similar platinum sensitivity was observed in this study. In a clinical study on taxane, regardless of previous platinum sensitivity in recurrent/metastatic patients with HNC, approximately half of the patients responded to docetaxel plus cetuximab combination treatment (20). Similarly, platinum-sensitive P-3 and P-5 PDXs were sensitive to taxane in this study. Second, the characteristic drug reaction rate in PDX models may affect the rapid drug response. Based on previous studies $(12,17)$, the drug responses in PDX models were relatively rapid and were observed about 2 weeks after drug administration where the corresponding patients were clinical responders. This rapid tumour size reduction may thus be a feature of drug responsiveness in PDX model. Despite limited sample size, these results suggest that drug responses in HNC PDXs reflect tumour response to the candidate drugs and that PDXs could be utilized for drug screening.

Because decrease of drug absorption and increase of drug efflux are common mechanisms of drug resistance, we focused on $\mathrm{ABC}$ transporters, which transport a large variety of drugs and mediate drug resistance. In this study, the expression of ABC transporters in three PDX models did not change even upon passage. However, the expression was increased by paclitaxel and cisplatin treatments in the P-3 PDX model, whereas the expression scores did not significantly change in P-2 and P-5 PDX models. The baselines of MDR-1 and MRP-2 expressions seems to be higher in P-5 tissue than in P-2 and P-3 tissues. Because P-5 patients and
PDXs were cisplatin responders and had higher expression of two ABC transporters, not only MDR-1/MRP-2 but also other $\mathrm{ABC}$ transporters might be involved in pharmacokinetics (4). In addition, the evasion of tumour cell apoptosis, other than drug efflux, may have an effect. Although the number of observations was limited and the association between cisplatin resistance and overexpression of $\mathrm{ABC}$ transporters was not determined, PDXs can be used in animal models to observe changes in target biomarker expression on drug administration. For example, ABC transporter-expressing PDXs might be used as an in vivo model to verify the effect of ABC transporter blocker.

Based on previous reports, we suggest three applications for PDX models in cancer research. First, they are a promising option for drug screening and biomarker development. The relationship between drug efficacy and molecular characteristics could be easily studied using PDX models, and previously, excellent biomarkers have been discovered for melanoma (21). Second, co-clinical trials could be performed. Co-clinical trials denote clinical trials that are conducted in parallel with PDX model experimentation. This type of trial provides a more useful platform than conventional trials to investigate biomarkers of drug response and resistance and can also be used to advance new drug development and clinical introduction (2). Third, these models could be used for precision medicine. Oncology research has evolved on the basis of the improved understanding of cancer genotypes and phenotypes, which has led to a new era of precision medicine. The patient could thus be treated with an appropriate drug that elicits the best response in corresponding PDXs (2). The increased use of PDXs will accelerate $\mathrm{HNC}$ research to investigate biomarkers and responses to new drugs.

\section{Acknowledgements}

Not applicable.

\section{Funding}

The present study was supported by Japan Society for the Promotion of Science KAKENHI (grant no. 17K16899) and a Research Grant from the Cancer Research Institute of Kanazawa University (grant no. 02-44).

\section{Availability of data and materials}

The datasets used and/or analysed during the current study are available from the corresponding author on reasonable request.

\section{Authors' contributions}

HM was involved in drafting the manuscript or revising it critically for important intellectual content. HM and $\mathrm{KE}$ made substantial contributions to conception and design, acquisition of data, and analysis and interpretation of data. $\mathrm{HM}$ and KE confirm the authenticity of all the raw data. YK aided in collecting patient samples and worked on the preparation of PDX models along with AN. MMK performed 
immunostaining. KI, TU, YN, SK and NW were involved in patient sample collection and contributed to analysis and interpretation of data. NG and TY contributed to the conception and design of the present study. TY worked on the final version of the manuscript. All authors discussed the results and commented on the manuscript. All authors read and approved the final manuscript.

\section{Ethics approval and consent to participate}

The present study complied with the Declaration of Helsinki and was approved by the Investigational Review Board of Kanazawa University (approval no. 2015-125; Kanazawa, Japan). All patients included in the present study provided written informed consent. Furthermore, all animal procedures were approved by the Ethical Committee of the Laboratory for the Animal Experiments, Graduate School of Medical Science, Kanazawa University (permit no. AP-173861; Kanazawa, Japan) and were performed in compliance with the guidelines of this committee.

\section{Patient consent for publication}

All patients included in the present study provided written informed consent for the publication of any associated data and accompanying images at the point of recruitment to the trial.

\section{Competing interests}

The authors declare that they have no competing interests.

\section{References}

1. DiMasi JA, Reichert JM, Feldman L and Malins A: Clinical approval success rates for investigational cancer drugs. Clin Pharmacol Ther 94: 329-335, 2013.

2. Jung J, Seol HS and Chang S: The generation and application of patient-derived xenograft model for cancer research. Cancer Res Treat 50: 1-10, 2018

3. Peng S, Creighton CJ, Zhang Y, Sen B, Mazumdar T, Myers JN Lai SY, Woolfson A, Lorenzi MV, Bell D, et al: Tumor grafts derived from patients with head and neck squamous carcinoma authentically maintain the molecular and histologic characteristics of human cancers. J Transl Med 11: 198, 2013.

4. Vrana D, Hlavac V, Brynychova V, Vaclavikova R, Neoral C, Vrba J, Aujesky R, Matzenauer M, Melichar B and Soucek P: $\mathrm{ABC}$ transporters and their role in the neoadjuvant treatment of esophageal cancer. Int J Mol Sci 19: 868, 2018.

5. Theile D, Gal Z, Warta R, Rigalli JP, Lahrmann B, Grabe N, Herold Mende C, Dyckhoff G and Weiss J: Antiproliferative efficacies but minor drug transporter inducing effects of paclitaxel, cisplatin, or 5-fluorourcil in a murine xenograft model for head and neck squamous cell carcinoma. Cancer Biol Ther 15: 436-442, 2014

6. Warta R, Theile D, Mogler C, Herpel E, Grabe N, Lahrmann B, Plinkert PK, Herolde-Mende C, Weiss J and Dyckhoff G: Association of drug transporter expression with mortality and progression-free survival in stage IV head and neck squamous cell carcinoma. PLos One 9: e108908, 2014.

7. Brierley JD, Gospodarowicz MK and Wittekind C: UICC TNM Classification of Malignant Tumours. 8th edition. Wiley Blackwell, New York, NY, 2017.

8. Tanabe H, Takada Y, Minegishi D, Kurematsu M, Masui T and Mizusawa H: Cell line individualization by STR multiplex system in the cell bank found cross-contamination between ECV304 and EJ-1/T24. Tiss Cult Res Commun 18: 329-338, 1999.
9. Cho YB, Hong HK, Choi YL, Oh E, Joo KM, Jin J, Nam DH, Ko YH and Lee WY: Colorectal cancer patient-derived xenografted tumors maintain characteristic features of the original tumors. J Surg Res 187: 502-509, 2014.

10. Klinghammmer K, Raguse JD, Plath T, Albers AE, Joehrens K, Zakameh A, Brzezicha B, Wulf-Goldenberg A, Keilholz U, Hoffmann J and Fichtner I: A comprehensively characterized large panel of head and neck cancer patient-derived xenografts identifies the mTOR inhibitor everolimus as potential new treatment option. Int J Cancer 136: 2940-2948, 2015.

11. Kimple RJ, Harari PM, Torres AD, Yang RZ, Soriano BJ, Yu M, Armstrong EA, Blitzer GC, Smith MA, Lorenz LD, et al: Development and characterization of HPV-positive and HPV-negative head and neck squamous cell carcinoma tumorgrafts. Clin Cancer Res 19: 855-864, 2013.

12. Stewart EL, Mascaux C, Pham NA, Sakashita S, Sykes J, Kim L, Yanagawa N, Allo G, Ishizawa K, Wang D, et al: Clinical utility of patient-derived xenografts to determine biomarkers of prognosis and map resistance pathways in EGFR-mutant lung adenocarcinoma. J Clin Oncol 33: 2472-2480, 2015.

13. Garrido-Laguna I, Uson M, Rajeshkumar NV, Tan AC, de Oliveira E, Karikari C, Villaroel MC, Salomon A, Taylor G, Sharma R, et al: Tumor engraftment in nude mice and enrichment in stroma-related gene pathways predicts poor survival and resistance to gemcitabine in patients with pancreatic cancer. Clin Cancer Res 17: 5793-5800, 2011.

14. Pergolini I, Morales-Oyarvide V, Mino-Kenudson M, Honselmann KC, Rosenbaum MW, Nahar S, Kem M, Ferrone CR, Lillemoe KD, Bardeesy N, et al: Tumor engraftment in patient-derived xenografts of pancreatic ductal adenocarcinoma is associated with adverse clinicopathological features and poor survival. PLoS One 12: e0182855, 2017.

15. Facompre ND, Sahu V, Montone KT, Harmeyer KM, Nakagawa $H$, Rustgi AK, Weinstein GS, Gimotty PA and Basu D: Barriers to generating PDX models of HPV-related head and neck cancer. Laryngoscope 127: 2777-2783, 2017.

16. Prince ME, Sivanandan R, Kaczorowski A, Wolf GT, Kaplan MJ, Dalerba P, Weissman IL, Clarke MF and Ailles LE: Identification of a subpopulation of cells with cancer stem cell properties in head and neck squamous cell carcinoma. Proc Natl Acad Sci USA 104: 973-978, 2007.

17. Yu J, Qin B, Moyer AM, Sinnwell JP, Thompson KJ, Copland JA III, Marlow LA, Miller JL, Yin P, Gao B, et al: Establishing and characterizing patient-derived xenografts using pre-chemotherapy percutaneous biopsy and post-chemotherapy surgical samples from a prospective neoadjuvant breast cancer study. Breast Cancer Res 19: 130, 2017.

18. Markman M, Rothman R, Hakes T, Reichman B, Hoskins W, Rubin S, Jones W, Almadrones L and Lewis JL Jr: Second-line platinum therapy in patients with ovarian cancer previously treated with cisplatin. J Clin Oncol 9: 389-393, 1991.

19. Harries M and Gore M: Part II: chemotherapy for epithelial ovarian cancer-treatment of recurrent disease. Lancet Oncol 3: 537-545, 2002.

20. Knoedler M, Gauler TC, Gruenwald V, Matzdorff A, Schroeder M, Dietz A, Jordan WO, Arnold D, Hennemann B, Hofele C, et al: Phase II study of cetuximab in combination with docetaxel in patients with recurrent and/or metastatic squamous cell carcinoma of the head and neck after platinum-containing therapy: A multicenter study of the Arbeitsgemeinschaft Internistische Onkologie. Oncology 84: 284-289, 2013.

21. Das Thakur M, Salangsang F, Landman AS, Sellers WR, Pryer NK, Levesque MP, Dummer R, McMahon M and Stuart DD: Modelling vemurafenib resistance in melanoma reveals a strategy to forestall drug resistance. Nature 494: 251-255, 2013.

This work is licensed under a Creative Commons Attribution-NonCommercial-NoDerivatives 4.0 International (CC BY-NC-ND 4.0) License. 